\title{
Mechanisms of Dendritic Integration Underlying Gain Control in Fly Motion-Sensitive Interneurons
}

\author{
ALEXANDER BORST* \\ MARTIN EGELHAAF** AND JÜRGEN HAAG* \\ borst@sunwan.mpik-tueb.mpg.de \\ Max-Planck-Institut für biologische Kybernetik, Spemannstrasse 38, D-72076 Tübingen, Germany
}

Received July 8, 1994; Revised October 18, 1994; Accepted (in revised form) November 4, 1994

Action Editor: J.P. Miller

\begin{abstract}
In the compensatory optomotor response of the fly the interesting phenomenon of gain control has been observed by Reichardt and colleagues (Reichardt et al., 1983): The amplitude of the response tends to saturate with increasing stimulus size, but different saturation plateaus are assumed with different velocities at which the stimulus is moving. This characteristic can already be found in the motion-sensitive large field neurons of the fly optic lobes that play a role in mediating this behavioral response (Hausen, 1982; Reichardt et al., 1983; Egelhaaf, 1985; Haag et al., 1992). To account for gain control a model was proposed involving shunting inhibition of these cells by another cell, the so-called pool cell (Reichardt et al., 1983), both cells sharing common input from an array of local motion detectors. This article describes an alternative model which only requires dendritic integration of the output signals of two types of local motion detectors with opposite polarity. The explanation of gain control relies on recent findings that these input elements are not perfectly directionally selective and that their direction selectivity is a function of pattern velocity. As a consequence, the resulting postsynaptic potential in the dendrite of the integrating cell saturates with increasing pattern size at a level between the excitatory and inhibitory reversal potentials. The exact value of saturation is then set by the activation ratio of excitatory and inhibitory input elements which in turn is a function of other stimulus parameters such as pattern velocity. Thus, the apparently complex phenomenon of gain control can be simply explained by the biophysics of dendritic integration in conjunction with the properties of the motion-sensitive input elements.
\end{abstract}

Keywords: motion detection, gain control, visual interneuron, dendritic integration

\section{Gain Control in Behavior and Motion-Sensitive Cells}

The optomotor response of the fly and other visually orienting animals has proven to be a useful tool for studying the primary mechanisms underlying the detection of visual motion. In the fly the behavior is usually measured with a torque meter by placing the experimental animal in the midst of a striped drum and rotating the cylinder around it. The animal then

*Present address: Friedrich-Miescher-Laboratorium der MaxPlanck-Gesellschaft, Spemannstrasse 37-39, D-72076 Tübingen, Germany.

**Present address: Centre for Visual Sciences, Research School of Biological Sciences, P.O. Box 475, Canberra City, A.C.T. 2601, Australia. displays a following response by generating a torque in the direction of image motion. Two essential processing steps are involved in transforming the retinal input signals into the torque response. First, motion information is extracted in parallel by arrays of local motion detectors. Many experiments have shown that their properties can be well approximated by the well-known correlation-type of motion detector (Götz, 1972; Buchner, 1984; Reichardt, 1987; Borst and Egelhaaf, 1989; Egelhaaf and Borst, 1993). Since, after local motion extraction, motion information is still represented in a spatially distributed form, some sort of integration is necessary as a second processing step in order to transform these distributed signals into a single steering signal. From a mathematical point of view, a linear summation would be the most simple form of spatial pooling. 


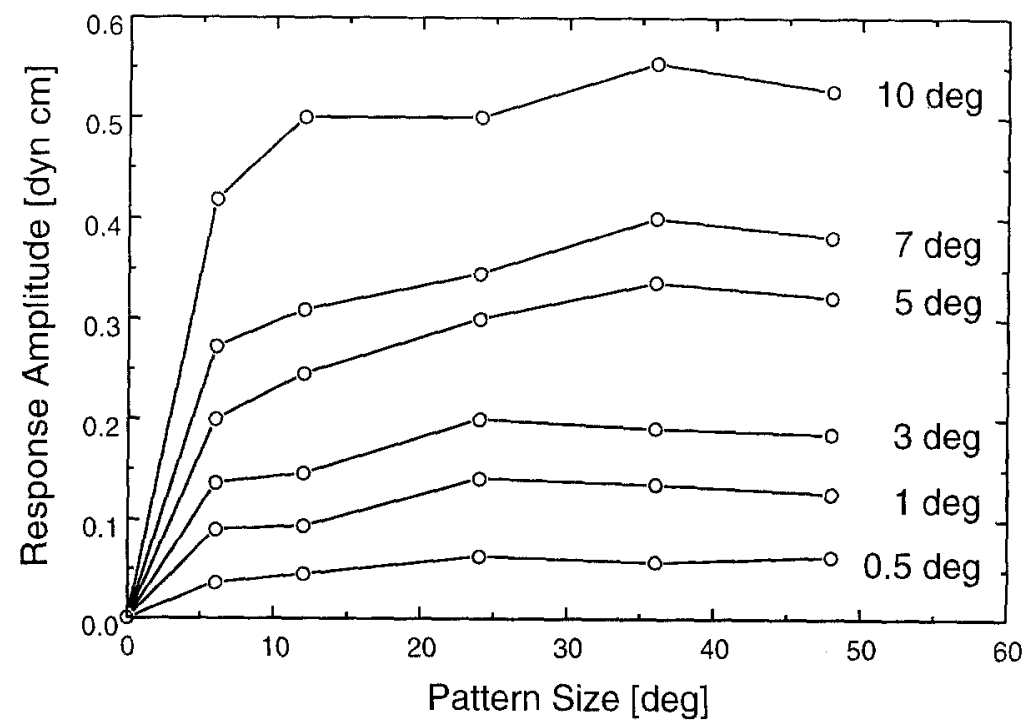

Fig. 1. Oscillation amplitude of the torque response of female houseflies Musca domestica as a function of the angular extent of an oscillating pattern $(0-48 \mathrm{deg})$ and the oscillation amplitude of the pattern $(0.5-10 \mathrm{deg})$. The oscillation frequency amounts to $2.5 \mathrm{~Hz}$. Thus, the maximum velocities reached during each experiment vary from $1.25 \mathrm{deg} / \mathrm{s}$ at $0.5 \mathrm{deg}$ to $25 \mathrm{deg} / \mathrm{s}$ at $10 \mathrm{deg}$ oscillation amplitude. The fly is suspended from a torque meter in the midst of a textured drum $(3 \times 3 \mathrm{deg}$ 'Julesz' pattern). Each data point is derived from 100 measurements. Data redrawn from (Reichardt et al. 1983).

However, the optomotor response does not depend on the size of the stimulus pattern in a linear way and, thus, linear summation is a rather poor description of spatial pooling. Instead, for a given amplitude of the oscillating stimulus pattern the response amplitude increases with increasing pattern size but reaches a more or less constant level when the pattern size exceeds about 12 degrees (Fig. 1). There are two noteworthy points: (i) The response increases less than linearly. This indicates some sort of saturation process inherent in spatial integration of the local motion detector signals. (ii) The saturation plateau depends on the actual stimulus parameters, in this case the velocity of the pattern. This excludes that the saturation observed for the different stimulus parameters is a simple output saturation. This finding is interesting in functional terms. Although the optomotor response of the fly is more or less independent of the spatial extent of the visual surround, it still is sensitive to changes in pattern velocity. This means, that the gain of the system is variable.

Motion-sensitive interneurons in the fly's optic lobes display similar response characteristics. In the rear part of the third visual ganglion, the so-called lobula plate, there resides a set of 50-60 individually identifiable interneurons (Fig. 2) which receive input from retinotopically arranged arrays of local motion-sensitive elements (Dvorak et al., 1975; Pierantoni, 1976; Hausen, 1976; Hausen et al., 1980; Hengstenberg et al., 1982; Hengstenberg, 1982; Borst and Egelhaaf, 1992). From various lines of evidence it is concluded that they are involved in the fly's course control (Geiger and Nässel, 1981; Geiger and Nässel, 1982; Hausen and Wehrhahn, 1983; Hausen and Wehrhahn, 1990; Heisenberg et al., 1978). In different representatives of these cells the phenomenon of gain control was studied (Hausen, 1982; Egelhaaf, 1985; Haag et al., 1992) leading to the following result (Fig. 3): (i) With increasing pattern size, the cellular response increases less than linearly tending to saturate at a given level (which may or may not be reached under the particular experimental conditions). (ii) For different velocities the cellular response approaches a different saturation level. Both statements are true for pattern moving in the cell's preferred direction, i.e. which excite the directionallyselective cells, as well as for pattern moving in the opposite direction, the so-called null direction which inhibits the cells.

Thus, the phenomenon of gain control is observed already at the level of the interneurons in the fly optic lobes. This suggests that the mechanism responsible for this phenomenon may involve these interneurons in some way or is presynaptic to them. 

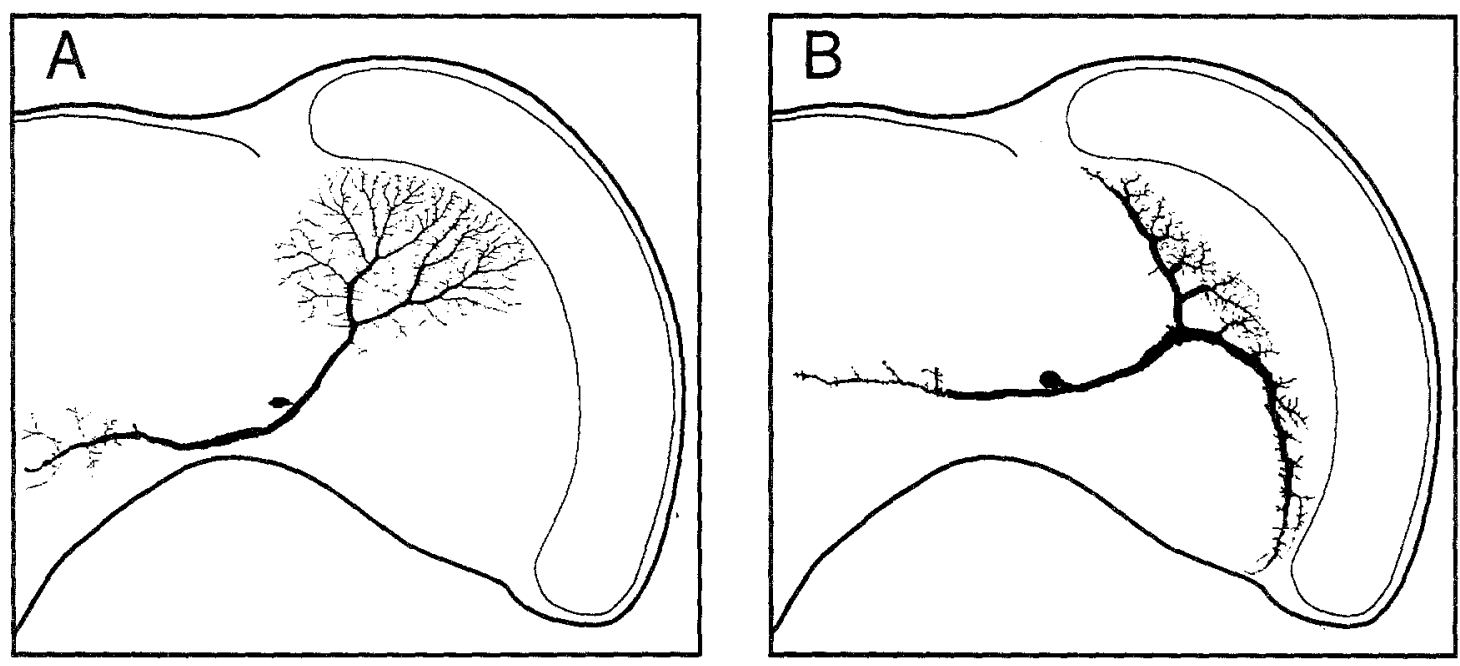

Fig. 2. Representatives of two different types of lobula plate motion-sensitive cells of the blowfly Calliphora erythrocephala. In A, one of the cells sensitive to horizontal motion (HSN-cells) is shown, in B there is a member of the cells sensitive to vertical motion (VS-cells). Cells were reconstructed from intracellular Cobalt injection. The schematic outline of the brain areas give a frontal view of the second and third visual neuropile and the central brain region.

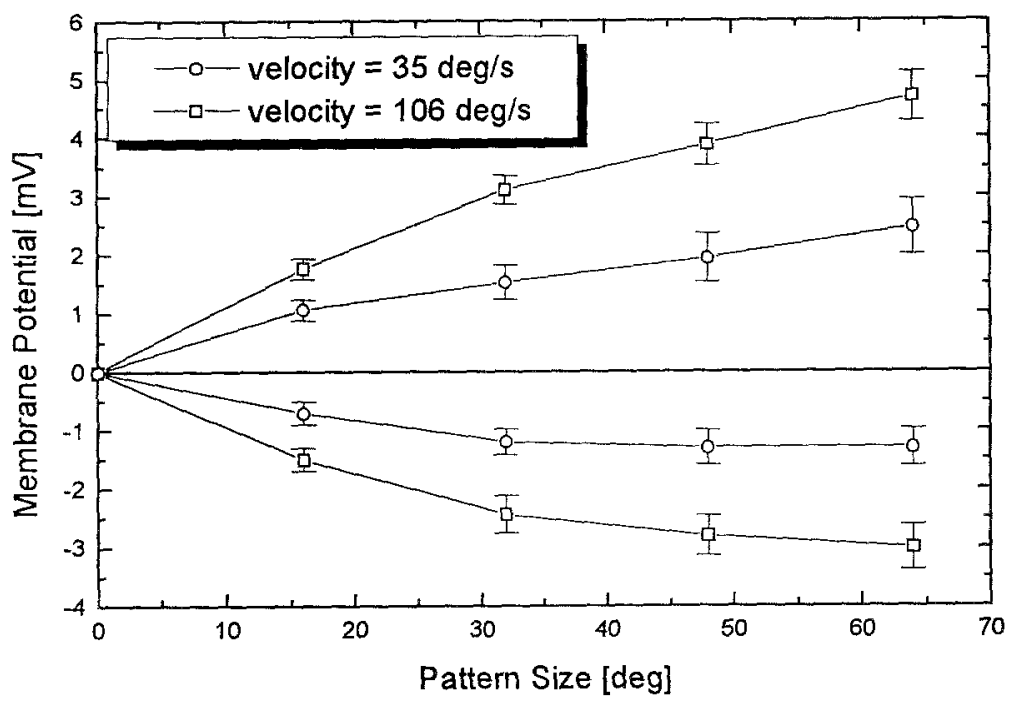

Fig. 3. Average response of lobula plate vertical cells (VS-cells, VS1-6) of female blowflies Calliphora erythrocephala in response to preferred (upper two curves) and null direction motion (lower two curves) at two different velocities. The pattern was displayed in a stimulus field of 40 deg horizontal extent and of variable vertical extent ('Pattern Size'). It consisted of a sine-grating of 9.3 deg spatial wavelength and $83 \%$ contrast. When activated the grating was moving for $2 \mathrm{~s}$. The data points represent the mean steady-state amplitude \pm SEM of the response obtained by averaging the membrane potential over the second stimulus half and subtracting the average membrane potential during the $1 \mathrm{~s}$ period preceeding the onset of the stimulus. Data are from 18 (velocity $=35 \mathrm{deg} / \mathrm{s})$ and $14($ velocity $=106 \mathrm{deg} / \mathrm{s})$ different VS-cells, respectively. Each neuron was tested with 1-7 sweeps per stimulus conditions. Data redrawn from (Haag et al. 1992).

\section{Models}

To account for the phenomenon of gain control Reichardt and colleagues proposed the following model circuit (Poggio et al., 1981; Reichardt et al., 1983) (Fig. 4): Local motion information is extracted from the changing retinal images by an array of 10cal motion detectors of the correlation type. Within 


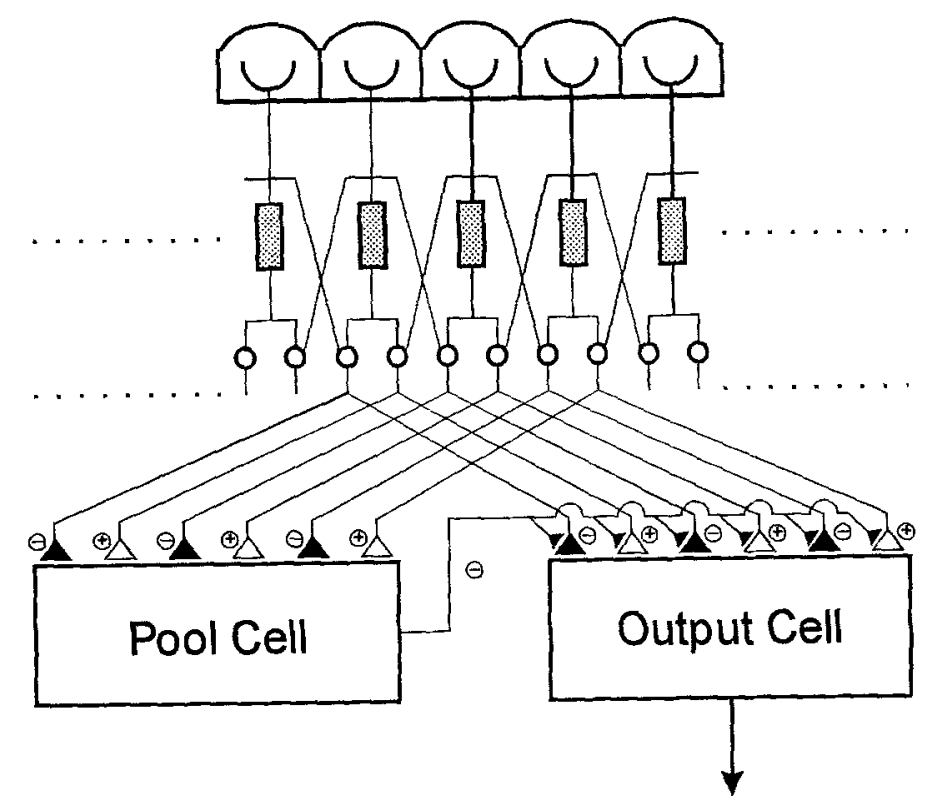

Fig. 4. Model of Reichardt et al. (Reichardt et al. 1983) to explain the phenomenon of gain control observed in flies at the behavioral (Fig. 1) and neuronal (Fig. 3) level. The output signals from an array of local correlation-type of motion detectors are spatially integrated by two large-field neurons, the pool- and the output cell. The pool cell acts on the input signals of the output cell through inhibition of the shunting type. By appropriate adjustment of the parameters, gain control is produced in the signal of the output cell.

each such local motion-sensitive unit, the input signal derived from one location in space is temporally lowpassed filtered and subsequently multiplied with the instantaneous signal from a neighboring location. At each location there exists a pair of such elements which are mirror images from each other and, thus, have opposite preferred directions. The output signals of the mirror-symmetrical elements impinge with excitatory and inhibitory synapses on the dendrites of two different large-field units, a 'pool cell' and the 'output cell' of the circuit.

While in this model the input synapses to the pool cell are assumed to have linear transmission characteristics, the input synapses to the output cell possess an expansive nonlinearity. As will be shown below, this is a crucial assumption for the circuit to produce gain control. The output signal of the pool cell is postulated to undergo a compressive nonlinearity before acting on the input signals of the output cell via presynaptic shunting inhibition. By appropriate choice of parameters, the signal of the output cell saturates with increasing pattern size but assumes different saturation plateaus for different pattern velocities just as was found in the experiments.

The model responses can be calculated in the following way (Reichardt et al., 1983): Assume that the output $x$ of each motion detector is proportional to pattern velocity, and that a pattern of given size excites $N$ such motion detectors. Spatial integration of these signals by the pool cell results in a signal proportional to $N x$ which, after saturation, is approximated by $(N x)^{q}$ with $q<1$. The input signals of the output cell are the same as of the pool cell, but become shunted by the pool cell's output signal $(N x)^{q}$. With a shunting coefficient $\beta$ representing the lumped leak conductances of the shunted cell, the input signals assume the value $x /\left(\beta+(N x)^{q}\right)$. Fed through an expansive nonlinearity with $n>1$ and integrated in a linear way, the signal in the output cell results in

$$
R=N x^{n} /\left(\beta+(N x)^{q}\right)^{n}
$$

If $n$ and $q$ are chosen such that $n q=1, R$ will saturate with increasing pattern size (for large values of $N$ ) at

$$
\lim _{N \rightarrow \infty} R=x^{n-1}
$$

Thus, the saturation plateau is solely set by the local pattern velocity $x$. This explanation of gain control critically depends on (i) the existance of a pool cell sharing its input elements with the output cell, and (ii) the exact values of $n$ and $q$ which determine the compressive 


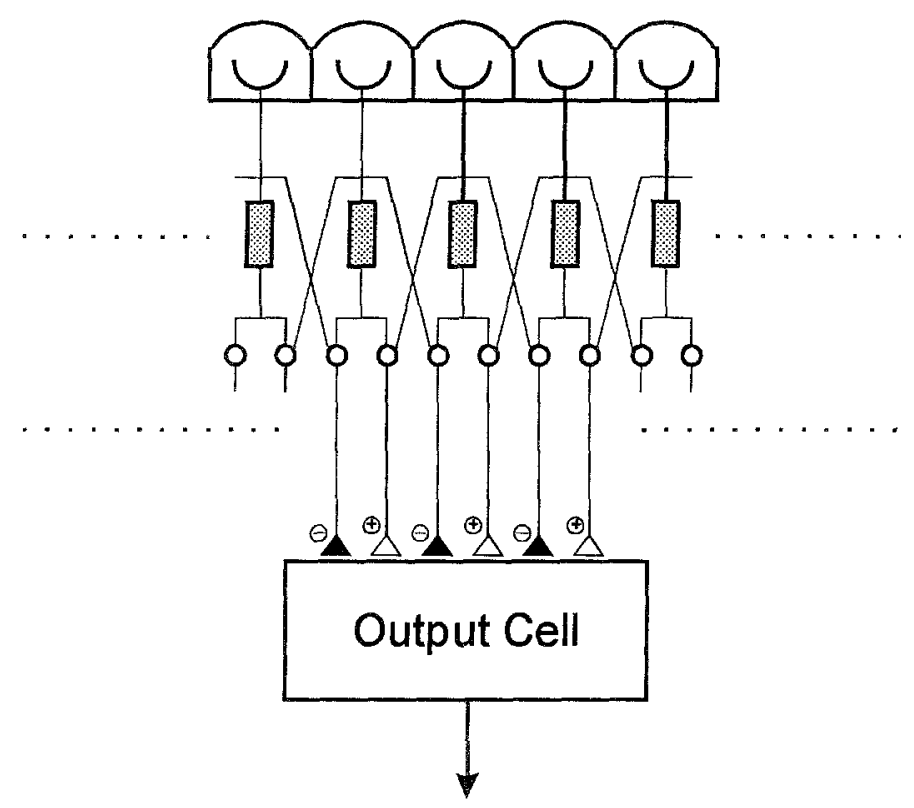

Fig. 5. Alternative model to explain gain control. In contrast to the original model (Fig. 3) no pool cell is needed that inhibits the output cell. Gain control comes about by the fact that (i) the excitatory and inhibitory subunits of each local motion detector have a low direction-selectivity thus being activated not only by motion in their preferred direction but also, though to a lesser extend, by motion in their null direction, and (ii) this activation ratio depends on stimulus parameters such as velocity. The simultaneous opening of excitatory and inhibitory ion channels leads to a mixed reversal potential the membrane potential in the dendrite of the output cell approaches with increasing pattern size.

and expansive nonlinear transmission characteristic between the pool cell and the input elements to the output cell, and between the input elements and the output cell, respectively.

However, gain control is displayed by all large-field cells of the fly lobula plate examined so far. Since these cells all have different preferred directions and different receptive field properties, one had to postulate for each of them a separate pool cell rendering this kind of circuit unlikely to be realized in the fly nervous system. To derive our alternative model (Fig. 5) we adopted a different approach which is simply based on the standard electrical equivalent circuit for a passive patch of membrane. Taking into account the properties of motion detectors which provide the input to the large-field cells, gain control is an inevitable consequence without making any additional assumptions.

The dendritic membrane potential $V$ is determined by the membrane conductances $g$ and reversal potentials $E$ in the following way:

$$
V=\left(E_{e} g_{e}+E_{i} g_{i}+E_{0} g_{0}\right) /\left(g_{e}+g_{i}+g_{0}\right)
$$

Here, $g_{e}, g_{i}$ and $g_{0}$ represent the excitatory, inhibitory and leak conductances, and $E_{e}, E_{i}$ and $E_{0}$ the respective reversal potentials. For the sake of simplicity, let us assume an isopotential dendrite of the large-field cell. By assuming that the excitatory and inhibitory conductances are proportional to the output signals of the local motion detectors and having $N$ such pairs activated (proportional to the pattern size), $V$ can be expressed as:

$$
V=\left(N E_{e} g_{e}+N E_{i} g_{i}\right) /\left(N g_{e}+N g_{i}+g_{0}\right)
$$

Here, $E_{0}=0, E_{e}>0$, and $E_{i}<0$. For large $N$ the postsynaptic potential $V$ approaches a value of

$$
\lim _{N \rightarrow \infty} V=\left(E_{e} g_{e}+E_{i} g_{i}\right) /\left(g_{e}+g_{i}\right)
$$

The output cell, thus, saturates with increasing pattern size at a value which is set by the respective excitatory and inhibitory reversal potentials and conductances. If motion in the cell's preferred direction only activates the excitatory input elements and, thus, $g_{i}$ were zero, there would be only a single saturation plateau $E_{e}$ for all stimulus conditions. However, correlation-type motion detectors behave differently, because a single subunit is not only activated during motion in its preferred direction but also, though to a smaller extent, during 
motion in the opposite direction (Egelhaaf et al., 1989; Borst and Egelhaaf, 1990). Thus, motion in the preferred direction of the excitatory input elements leads also to an activation of the inhibitory input elements. If we express the inhibitory conductance $g_{i}$ as a fraction $c$ of the excitatory conductance $g_{e}$, we can rewrite expression (3) in the following way:

$$
V=N g_{e}\left(E_{e}+c E_{i}\right) /\left(N g_{e}(1+c)+g_{0}\right)
$$

For large values of $N, V$ now approaches

$$
\lim _{N \rightarrow \infty} V=\left(E_{e}+c E_{i}\right) /(1+c)
$$

The postsynaptic membrane potential, thus, does not saturate at the excitatory reversal potential for large pattern sizes, but at a potential between the excitatory and inhibitory reversal potentials. This is set by (i) the absolute values of these reversal potentials, and (ii) by the activation ratio $c$ of the respective input elements. The saturation value of the postsynaptic membrane potential $V$ for large pattern sizes can be further simplified by the assumption that the resting potential of the large-field cell lies approximately in the middle between the reversal potentials of the excitatory and inhibitory inputs: $E_{i}=-E_{e}$. It then becomes:

$$
\lim _{N \rightarrow \infty} V=E_{e}(1-c) /(1+c)
$$

To fully understand gain control just on the basis of dendritic integration of local motion detectors one needs to formulate the relationship between the activation ratio $c$ of opponent motion detectors and stimulus properties like pattern velocity, contrast and spatial wavelength.

Consider a sine-grating with a mean luminance $I$, a modulation $\Delta I$ and spatial wavelength $\lambda$. The grating is moving at a velocity $v$ in the preferred direction of a motion detector with two inputs separated by an angle $\Delta \varphi$ (the 'sampling base') and equipped with a low-pass filter with time-constant $\tau$ (Fig. 6) (Reichardt, 1961; Reichardt, 1987; Buchner, 1984; Borst and Egelhaaf, 1993). With the circular frequency $\omega=2 \pi v / \lambda$, the input signals $S_{1}$ and $S_{2}$ to the motion detector are:

$S_{1}=I+\Delta I \sin (\omega t) ; \quad S_{2}=I+\Delta I \sin (\omega(t-\Delta t))$

Since the time delay $\Delta t$ equals $\Delta \varphi / v$ we can rewrite $S_{2}$ as $I+\Delta I \sin (\omega t-2 \pi \Delta \varphi / \lambda)$. Denoting $A(\omega)$ and $\varphi(\omega)$ as the amplitude and phase response of the lowpass filter in dependence of the circular frequency $\omega$ and $S^{\prime}$ as the response of the low-pass filter to the input function $S$ the signals being multiplied in the left

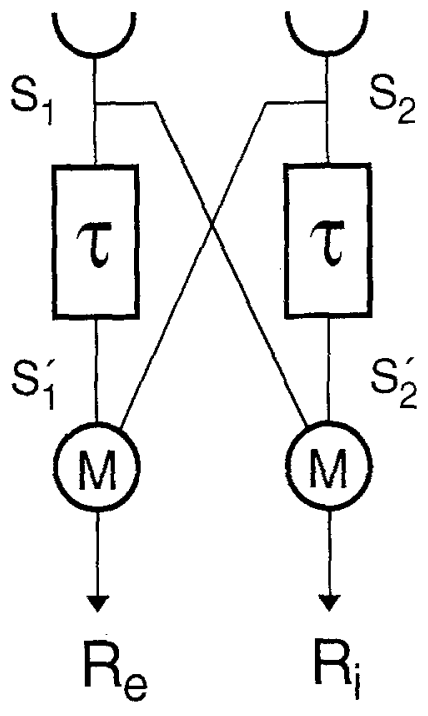

Fig. 6. Correlation-type motion detector (Reichardt, 1961; Reichardt, 1987). At each retinal location the luminance values changing over time are processed in two mirror-symmetrical subunits. Each subunit receives input from two adjacent retinal locations, one of which is filtered and subsequently multiplied with the unfiltered signal. The resulting output signals respond to pattern motion in a directionally selective way.

detector subunit (see Fig. 5) become

$$
\begin{aligned}
& S_{1}^{\prime}=I+A(\omega) \Delta I \sin (\omega t+\phi(\omega)) \quad \text { and } \\
& S_{2}=I+\Delta I \sin (\omega t-2 \pi \Delta \varphi / \lambda)
\end{aligned}
$$

The product of $S_{1}^{\prime}$ and $S_{2}$ contains four additive terms two of which are time dependent with an average value of zero, and two time independent terms. Only the latter have to be taken into account if the considerations are restricted to the steady-state response. Applying simple trigonometric rules the response $R_{e}$ of one motion detector can be calculated as (Reichardt, 1961; Reichardt, 1987; Buchner, 1984; Borst and Bahde, 1986; Egelhaaf et al., 1989):

$$
R_{e}=I^{2}+\Delta I^{2} A(\omega) \cos (2 \pi \Delta \varphi / \lambda+\phi(\omega)) / 2
$$

The response of its mirror-symmetrical counterpart to pattern motion in the same direction becomes:

$$
R_{i}=I^{2}+\Delta I^{2} A(\omega) \cos (2 \pi \Delta \varphi / \lambda-\phi(\omega)) / 2
$$

The activation ratio $c$ of the excitatory and inhibitory input, thus, depends on local pattern parameters like the velocity $v$ and spatial wavelength $\lambda$ which both determine the circular frequency $\omega$ as well as the mean luminance $I$ and the modulation $\Delta I$. This predicts 
that if one changes just one of these parameters like e.g. pattern velocity, different saturation values should be assumed for large pattern sizes. Thus, gain control can be explained without any further assumptions. It just relies on the basic biophysical properties of nerve cell membranes and the well-studied properties of correlation-type of motion detectors.

To make quantitative predictions we can go one step further by taking into account the finding that the luminance is unlikely to be fully represented in the input lines of the local motion detector (Egelhaaf et al., 1989). If we assume the mean luminance $I$ to be small compared to the other expression in Eqs. (6) and (7), the activation ratio $c$ can be approximated by:

$c=\cos (2 \pi \Delta \varphi / \lambda-\varphi(\omega)) / \cos (2 \pi \Delta \varphi / \lambda+\phi(\omega))$

This expression has some interesting implications. It says that changes in pattern contrast should have no influence on the saturation level the system assumes for large pattern sizes. In contrast, changes in pattern velocity and spatial wavelength will affect the saturation level according to the precise values of each parameter. For further interpretations of the Eq. (13), however, care has to be taken that neither one of the variables $R_{e}$ and $R_{i}$ assumes negative values since then they loose their interpretation as conductances.

\section{Simulations}

The calculations of the model response as presented above involve two simplifications: (i) The consideration of only steady-state responses of the movement detector and the integrating unit, and (ii) the assumption of an isopotential dendrite of the output cell. To investigate to which extent these simplifying assumptions affect the conclusions drawn above, we simulated the model performance without these restrictions on a computer (Mac Quadra 900, using MPW Pascal as programming language). The model comprises an array of 16 pairs of motion detectors and an integrating cell consisting of 43 compartments 16 of which (the dendritic compartments) receive excitatory and inhibitory input from the motion detector array (Fig. 7).

The topology of the output neuron was chosen such that all dendritic compartments have an equal electrotonic distance from the integrating compartments which gives rise to a one dimensional string of compartments considered as the axon of the cell. All dendritic as well as axonal compartments are con- nected with each other through equal length conductances and possess no voltage activated conductances. Leak and length conductance are chosen such that each compartment has an approximate electrotonic length of 0.07 . The exact values used in the simulation for each compartment are as follows: (i) equal leak conductance of 0.05 for all compartments, (ii) a length conductance of 10 for axonal and 1 for dendritic compartments, and (iii) a value of 0.01 for the capacitance. Assuming that an axonal compartment were a cylinder with $20 \mu$ diameter and $100 \mu$ length, and setting the time unit to $10 \mathrm{~ms}$, these values would translate to a specific membrane resistance $R_{m}$ of $1256 \Omega \mathrm{cm}^{2}$, a specific internal resistivity $R_{i}$ of $31.4 \Omega \mathrm{cm}$, a specific membrane capacitance $C_{m}$ of $1.6 \mu \mathrm{F} / \mathrm{cm}^{2}$ and a membrane time constant $\tau_{m}$ of about $2 \mathrm{~ms}$. Pattern motion is simulated by rotating the pattern values within the array at each time step by an amount according to the actual velocity. Low pass filtering is accomplished by use of the discretized form of the differential equation of a firstorder low-pass filter $y(t+1)=1 / \tau(x(t)-y(t))+$ $y(t)$. According to our findings that the mean luminance is represented to only a small extent at the input to the motion detectors the effective contrast of the pattern $\Delta I / I$ can well assume values larger than 1 . By this way, however, the time dependent output signals of the local motion detectors can assume negative values as well which cannot be readily translated into membrane conductances. Instead of clipping the negative parts we account for this by adding the negative parts of the otherwise excitatory conductances to the inhibitory ones and vice versa. In summary, the whole simulation is represented mathematically as a set of linear $1 \mathrm{st}$ order differential equations and is solved by standard numerical techniques (Segev et al., 1989).

The simulations results are shown in Fig. 8. For all runs, the mean luminance is chosen to be 0.1 , the spatial wavelength of the pattern is $32 \mathrm{deg}$, the sampling base equals 4 deg and the detector time constant $\tau_{D}$ is 2 time units. The parameters varied are the size of the pattern, the pattern velocity and the pattern contrast. The size of the pattern is varied from 4 to $64 \mathrm{deg}$. The velocity is varied between 1 and 16 deg per time unit while the luminance modulation is held at a value of 0.4 . To change the contrast, the luminance modulation is varied from 0.1 to 1.6 while the velocity is held constant at a value of 4 deg per time unit. If again one time unit is considered to be equal to $10 \mathrm{~ms}$, the detector time constant $\tau_{D}$ amounts to $20 \mathrm{~ms}$ and the velocities range from 100 to $1600 \mathrm{deg} / \mathrm{s}$ resulting in temporal frequencies between 3 and $50 \mathrm{~Hz}$. This range covers 

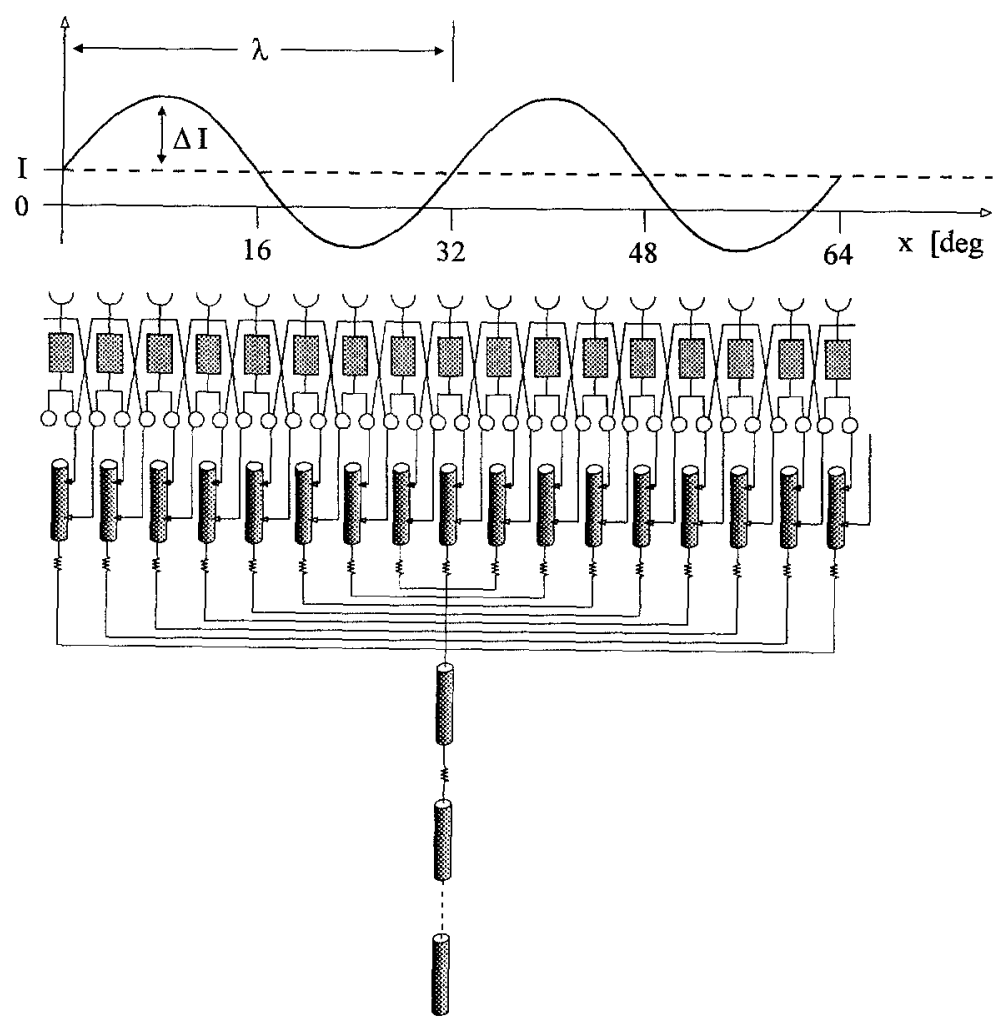

Fig. 7. Outline of the model simulation. A spatial sine grating (wavelength $\lambda=32 \mathrm{deg}$, mean luminance $I=0.1$, modulation $\Delta I=0.8$ ) is moved in front of an array of 16 pairs of correlation-type of motion detectors the inputs of which are separated by the sampling base $\Delta \varphi=4$ deg. Within each of them the light intensity as measured at one location in visual space is filtered by a first-order low-pass filter (time constant $\tau=2$ time units) and subsequently multiplied with the instantaneous light intensity as measured at the neighboring location. This operation is done twice in a mirror-symmetrical fashion at each location. While the output signals of the detectors tuned for rightward motion control excitatory conductances of the integrating neuron, those of the leftward tuned detectors control inhibitory conductances. The integrating neuron is split into 43 isopotential compartments. The membrane conductance of the 16 dendritic compartments is increased through synaptic input from the local motion detectors in the way specified above with reversal potentials of $+30 \mathrm{mV}$ and $-30 \mathrm{mV}$ (relative to resting potential) for the excitatory and inhibitory synapses, respectively. The compartments are connected with each other as shown in the figure and contain no voltage-gated conductances.

the maximum steady-state detector response which is expected to be at a temporal frequency of $1 /\left(2 \pi \tau_{D}\right)$ (Borst and Bahde, 1986) and, hence, at about $8 \mathrm{~Hz}$. The dynamics of the response are completely determined by the value of the detector time constant $\tau_{D}$ and not by the membrane time constant $\tau_{m}$ of the integrating neuron. Within the part of the parameter space taken into account here the model behaves as is expected from the calculations presented above.

The response of the output cell as a function of pattern size saturates at different levels when the pattern moves at different velocities (Fig. 8). Furthermore, for a given pattern size, the model exhibits a peaked velocity dependence as is expected from motion detector theory (Reichardt, 1961; Götz, 1972). A quan- titative inspection of the size-dependence at different stimulus velocities reveals the following: If a saturation curve of the form $y=A x /(x+b)$ is fitted to the simulation results, different values of $A$ are obtained for different velocities while the values of $b$ remain rather constant. Since $A$ determines the value the function will reach for large $x$ values, and $b$ determines the value of $x$ at which half-maximum response is obtained, these results indicate that the pattern velocity mainly affects the saturation plateau and not the shape of the saturation curve. This is in accordance with the approximations presented above. When instead of pattern velocity the contrast of the pattern is varied the response always approach the same saturation level $A$ and mainly the shape $b$ of the curve is altered. This 

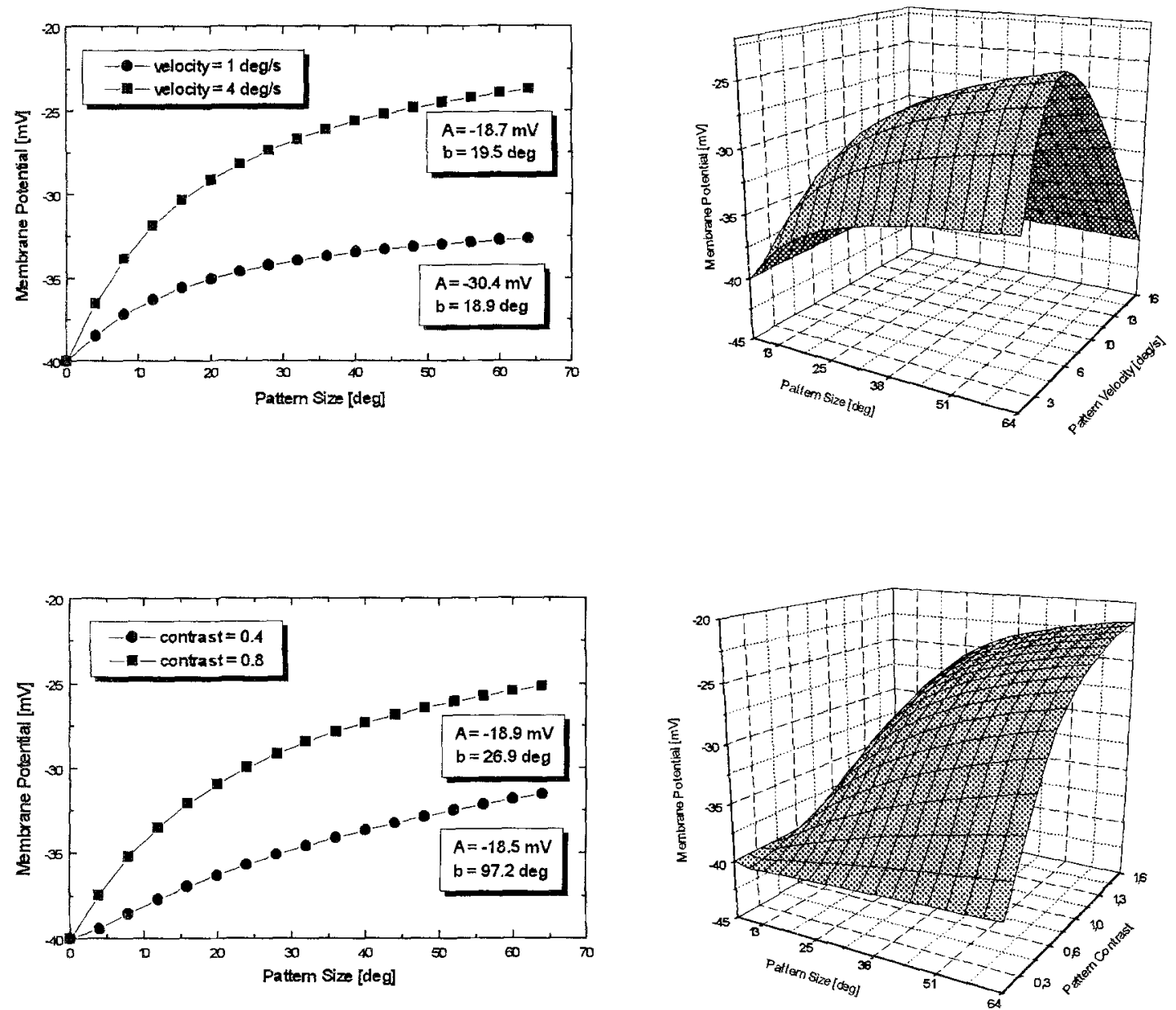

Fig. 8. Result of model simulations. An artificial nerve cell receiving its synaptic input from an array of correlation-type of motion detectors was stimulated with pattern motion of variable size. While in the upper part of the figure the velocity of the pattern is varied additionally, the contrast of the pattern is varied in the lower part. Two size-dependences at different simulus parameters are shown to the left. The numerical values of parameters $A$ and $b$ shown in the insets next to each graph represent the result of fitting the simulation data to a saturation function of the form $R=A * x /(x+b)$. To the right, the full response is shown as a function of both pattern size and pattern velocity or contrast, respectively.

again is in accordance with the calculations presented above.

\section{Experimental Evidence}

The model to explain gain control presented here builds critically upon three assumptions: (i) Largefield motion-sensitive cells receive excitatory and inhibitory input from local motion-sensitive elements with opposite preferred directions, (ii) These elements are not completely directionally selective, i.e. they also respond to motion in their anti-preferred or null direction, and (iii) The activation ratio of their responses to preferred and null direction motion changes with pat- tern velocity. In the following experimental evidence for these assumptions will be summarized.

Figure 9 (A and B) shows the response of a motionsensitive large-field cell (VS-cell) of the fly optic lobe to motion in its preferred and null direction, respectively. As can be seen, the cell depolarizes in response to preferred direction motion, and it hyperpolarizes when the pattern moves in the opposite direction. This can be explained in principally two different ways: Either the cell receives input from just one set of motion-sensitive elements the transmitter release of which is modulated around some spontaneous level, or the cell receives input from two types of motion-sensitive elements each one of which is activated by motion in the respective direction. In the first case, there should exist only one 


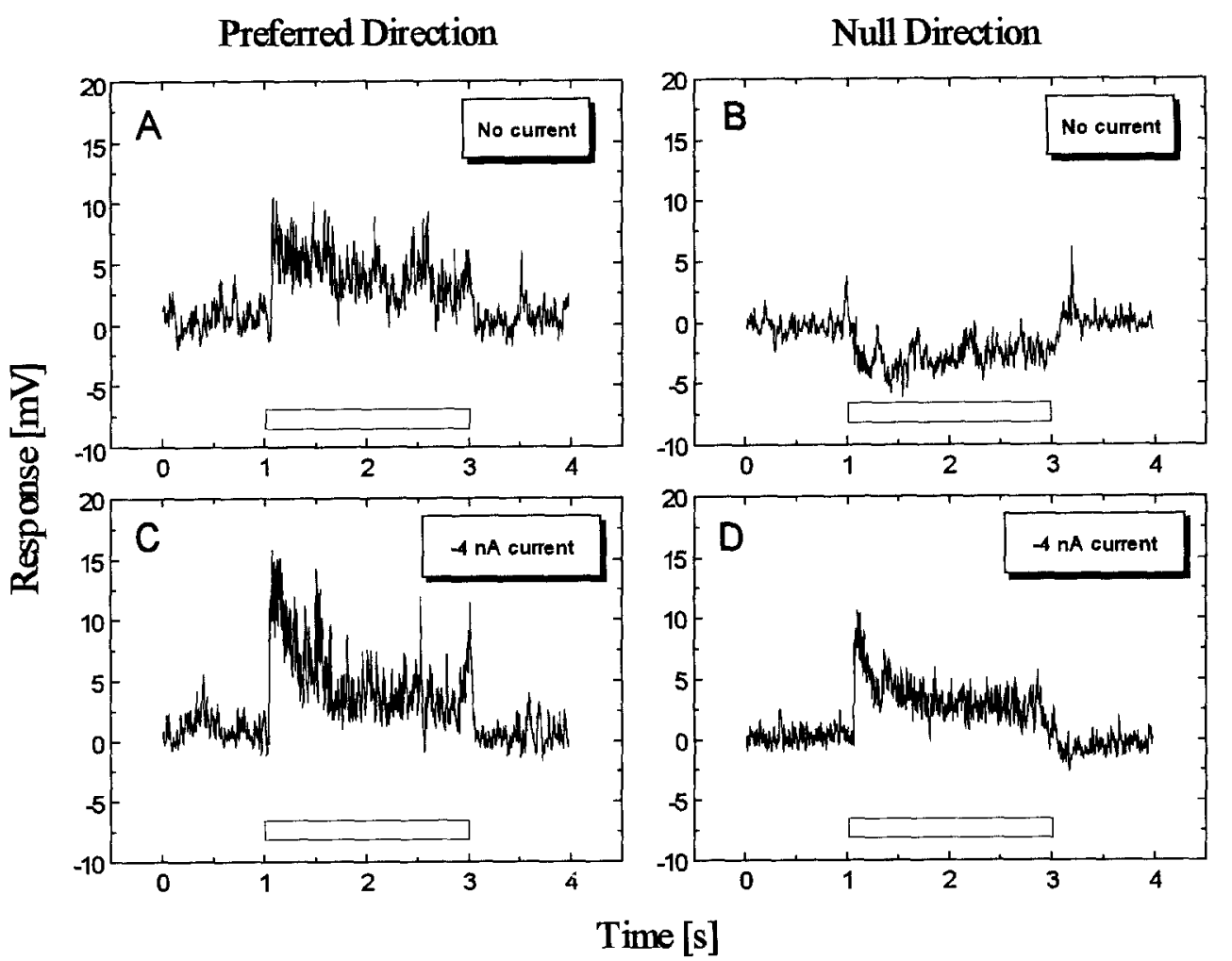

Fig. 9. Intracellular recordings from the dorsal part of the main dendrite of a motion-sensitive vertical cell (VS3 cell) of a female blowfly Calliphora erythrocephala while the cell was stimulated with either downward motion (preferred direction, A and C) or upward motion (null direction, B and D) displayed within the dorsal part of its receptive field. In A and B no current was applied while in C and D 4 nA of hyperpolarizing current was injected into the cell during motion stimulation. The membrane potential before motion onset is set arbitrarily to zero. Note the reversal of the null direction response in $\mathrm{D}$.

postsynaptic reversal potential, while in the latter two different reversal potentials are expected, one excitatory and one inhibitory. The obvious experiment to do is to modify in some way the postsynaptic membrane potential and try to flip one of the responses. If the other response changes its sign at the same time, there exists only one reversal potential, if the sign of the other response remains unaffected we are dealing with two different reversal potentials and, thus, two types of input channels. The experiment has been done in two ways. In one set of experiments performed on another type of horizontal cell, the so-called H1-cell, the visual scene was split in two areas, a test area in which the pattern moved in either the cell's preferred or null direction, and a surround, in which the pattern either moved in the cell's preferred direction or did not move at all. The latter stimulus was used to shift the dendritic membrane potential. When the cell was depolarized preferred and null direction stimuli within the test area released responses of opposite sign, while when the cell was at rest, both kinds of stimuli led to depolarizing responses.
Thus, the null response could be flipped independently of the response to preferred direction motion (Borst and Egelhaaf, 1990). The other experiment is shown in Fig. 9 (C and D). We manipulated the postsynaptic membrane potential of a so-called VS-cell by current injection into the dorsal part of main dendrite of the cell while stimulating the cell with pattern motion which affects just this synaptic area. This is because these cells receive motion information from the outside world in a strictly retinotopic fashion so that motion in the dorsal part of the receptive field activates synapses located on the dorsal dendrite, whereas motion in the ventral part of the receptive field activates synapses located on the ventral dendrite. The retinotopic input organization of the fly large-field cells although for long been assumed based on anatomical criteria has only recently been shown in physiological experiments by visualizing postsynaptic calcium influx actived by locally restricted motion stimulation (Borst and Egelhaaf, 1992). Thus, the electrode placement within the area of the dorsal dendrite should allow at least for limited 


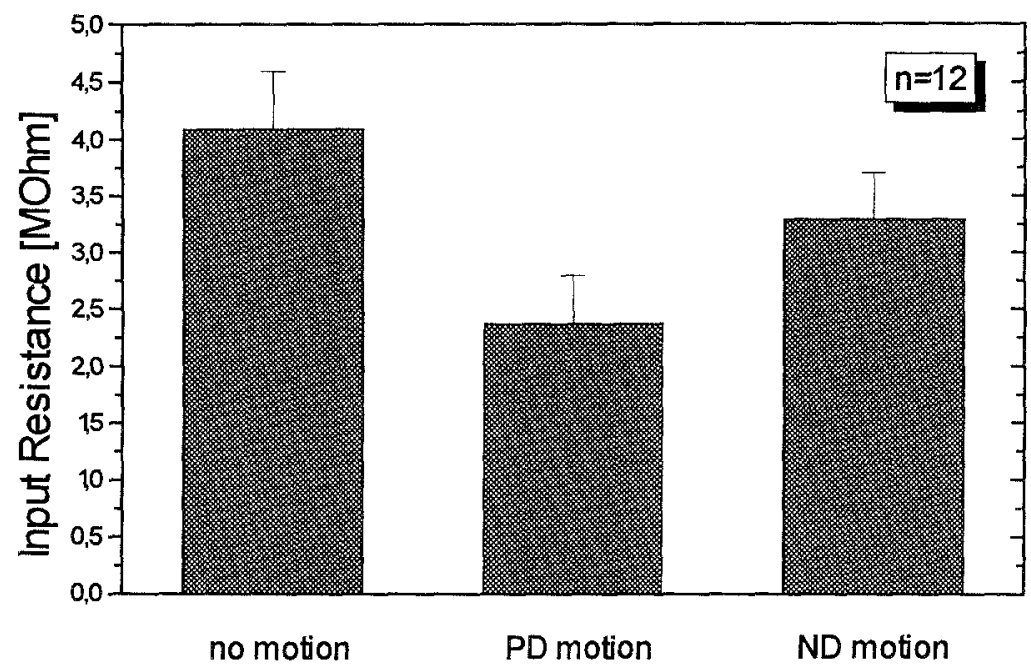

Fig. 10. Measurements of input resistance on lobula plate cells sensitive to horizontal motion (HS-cells) of female blowflies Calliphora erythrocephala during three different stimulus conditions: While the pattern is at rest (no motion), while the pattern is moving from the front to the back of the fly (preferred direction or PD motion) and while the pattern is moving in the opposite direction (null direction or ND motion). Data are mean \pm SEM obtained from 12 different cells. Note that both during preferred and null direction motion the input resistance drops.

control of the postsynaptic potential. Figure $9 \mathrm{C}$ and $D$ shows what happens when a hyperpolarizing current of $4 \mathrm{nA}$ is injected: When the cell is stimulated by preferred direction motion, it still responds with a depolarization of its membrane potential. However, the response to null direction motion is flipped: Instead of being hyperpolarized as is the case under normal conditions (Fig. 9B), the cell now responds also with a depolarization relative to its membrane potential before motion stimulation. These two experiments (Borst and Egelhaaf, 1990, and the one shown in Fig. 9) provide direct evidence for two types of inputs to this large-field cell, i.e. excitatory and inhibitory.

Further evidence for this particular input organization of the motion-sensitive large-field cells is provided by experiments where the input resistance of the cell is measured while the pattern is at rest, moving in the preferred direction or in the null direction. If there were only one type of input element involved in this response, it should increase its transmitter release under one circumstance, and decrease it during the other. This would lead to postsynaptic channels being opened in one case and channels being closed in the other case. Therefore, the input resistance of the cell should increase and decrease, depending on the direction of motion. If there were two types of input involved, and, thus, an increased number of ionic channels opened during both null- and preferred direction motion, the input resistance is expected to drop both times.
The experiment shown in Fig. 10 presents the respective data collected from 12 different horizontalsensitive cells, the so-called HS-cells. When the pattern is at rest, the input resistance as measured in the axon by injection of $2 \mathrm{nA}$ of hyperpolarizing current is around $4 \mathrm{M} \Omega$. When the pattern moves from the front to the back of the fly's eye, the cell's preferred direction, the input resistance drops to values well below $3 \mathrm{M} \Omega$. When the pattern is moving in the opposite direction, the input resistance also drops, though not to as small values as during preferred direction motion. Again, this experiment clearly speaks in favor of the two-types of input hypothesis and against the assumption that there is only one type of fully directionally selective input. It also is in quantitative accordance with similar experiments performed by Gilbert (Gilbert, 1991) who observed on the same type of motion-sensitive largefield cells a drop of input resistance as measured in the axon of about $1.9 \mathrm{M} \Omega$ during preferred direction motion and of about $1 \mathrm{M} \Omega$ during null direction motion. The quantitative difference between the drop of input resistance during both types of motion stimuli can be readily explained by additional voltage-gated ion channels activated only during preferred direction motion and not during null direction motion (Haag et al., 1993).

As mentioned above there are other assumptions our present explanation of gain control relies on. Beside the existence of separate excitatory and inhibitory input signals to the large-field neurons of the lobula plate, 
the second critical assumption is that these motionsensitive units are not perfectly directionally selective. By applying the chloride channel blocker picrotoxin to the fly hemolymph and recording extracellularly the spike frequency of the large-field motion sensitive neuron $\mathrm{H} 1$, a rise of the cell's response to preferred direction motion as well as of its response to null direction motion was observed (Schmid and Bülthoff, 1988; Egelhaaf et al., 1990). Moreover, the cell's response to flicker stimuli increased, too. This finding is interpreted as a consequence of blocking the cell's inhibitory input channels by picrotoxin. If the opponent input elements had an absolute direction selectivity, no change in the response to preferred direction motion were expected, and the null direction response should be completely abolished which is in contrast to experimental results. Fourier analysis of the H1 cell's response to a drifting sine-grating provided further evidence that the site of action of picrotoxin is indeed the subtraction of opponent input signals and not the nonlinear interaction between signals originating from neighboring photoreceptors (Egelhaaf et al., 1990). The third assumption concerns the way by which the direction selectivity of the individual motionsensitive input elements depends on the velocity of the moving pattern. Since these input elements have not been identified so far, no data exist and the argument will have to be based on the behavior of the large-field cell after application of the chloride channel blocker picrotoxin. These experiments are currently under way.

\section{Discussion}

The explanation of gain control presented here is based on the biophysics of dendritic integration and the properties of biological motion detectors. On the dendritic side, the model postulates synaptic saturation as being responsible for the response plateau. On the input side, the model postulates the simultaneous action of excitatory and inhibitory motion detectors the activation ratio of which change with velocity. Interestingly, the model only involves passive membrane properties of the integrating neuron without the participation of voltage-gated ion channels. This requires that, indeed, the passive membrane properties of the dendrite are such that changes in input resistance generated by motion input at one location influence other dendritic locations as well. The important membrane parameters determining the electrotonic distance between neighboring dendritic areas like specific length and membrane resistance are currently being investigated. Preliminary results suggest that, qualitatively, these values are within the order of magnitude to allow for such interactions between the different dendritic areas making the interpretation of the saturating size dependence of the optomotor response as a synaptic saturation plausible (Haag and Borst, 1994). Thus, future work will involve compartmental model simulations such as the one shown in Fig. 7 but which, in addition, take into account (i) the detailed anatomy of the fly tangential cells, (ii) their experimentally determined passive and active membrane properties, and (iii) the motion induced changes in input resistances as shown in Fig. 10.

Interestingly, Carandini and Heeger (1994) proposed a somewhat similar mechanism to account for the response properties of orientation selective cells in primate visual cortex. In their model, the authors propose that the response of each cell is determined by a linear synaptic input from LGN neurons and a shunting inhibition 'normalization' input from the summated signals of other cells (the 'pool') receiving similar input from the LGN. This model can explain nonlinear response characteristics such as the fact that the response amplitude as a function of stimulus contrast saturates, and that these contrast functions when measured at different stimulus orientations saturate at different levels.

Of course, alternatively to the mechansim proposed herein, a saturation characteristic could as well be the consequence of voltage-gated processes such as an outward rectification by $K$-currents. In non-spiking local interneurons of the locust such $K$-currents, amongst other voltage-gated currents, have been shown to be responsible for gain control observed within these neurons (Laurent, 1993). Calcium imaging experiments in the fly revealed that following synaptic stimulation calcium is accumulating in the dendrites through the activation of voltage-gated calcium channels (Borst and Egelhaaf, 1992; Egelhaaf and Borst, in prep.). Thus, there exists at least one voltage-gated membrane current the function of which, however, is not known so far. However, the fact that gain control acts for hyperpolarizing null direction motion stimuli as well as for depolarizing preferred direction motion stimuli makes it highly unlikely that both could be due to a current which is activated only by depolarization. Furthermore, the existence of several saturation plateaus for different velocities cannot be readily explained by such a hypothesis.

The mechanism proposed herein makes a number of predictions which can tested in future experiments. For 
instance, if the mean luminance is indeed represented to only a negligible extent in the motion detector input signals as proposed in earlier studies (Egelhaaf and Borst, 1989; Egelhaaf et al., 1989), then gain control should not be very pronounced for different contrasts (see also Fig. 8). The response of the integrating cell should approach similar saturation levels with increasing pattern size. Slicing the two-dimensional function $R=f$ (Size, Contrast) in the orthogonal direction, the prediction is that using different stimulus extents different contrast dependences should be obtained but velocity dependence should remain unaltered in shape. Of course, these predictions only hold for the mathematically ideal model which does not contain any voltagegated currents. It remains to be seen in which way such currents affect the predictions quantitatively.

\section{Acknowledgments}

We are grateful to K.G. Götz for his generous support of the project and for many helpful discussions, to T. Brotz and $S$. Single for critically reading the manuscript and to M. Bauch and A. Wildemann for technical assistance.

\section{References}

Borst A and Bahde S (1986) What kind of movement detector is triggering the landing response of the housefly? Biol. Cybern. 55:59-69.

Borst A and Egelhaaf M (1989) Principles of visual motion detection. Trends Neurosci. 12:297-306.

Borst A and Egelhaaf M (1990) Direction selectivity of fly motionsensitive neurons is computed in a two-stage process. Proc. Natl. Acad. Sci. USA 87:9363-9367.

Borst A and Egelhaaf M (1992) In vivo imaging of calcium accumulation in fly interneurons as elicited by visual motion stimulation. Proc. Natl. Acad. Sci. USA 89:4139-4143.

Borst A and Egelhaaf M (1993) Detecting visual motion: Theory and models. In: FA Miles and J Wallman, eds. Visual motion and its role in the stabilization of gaze. Amsterdam, London, New York, Tokyo: Elsevier, pp. 3-27.

Buchner E (1984) Behavioral analysis of spatial vision in insects. In: MA Ali, ed. Photoreception and vision in invertebrates. New York, London: Plenum Press, pp. 561-621.

Carandini M and Heeger DJ (1994) Summation and division by neurons in primate visual cortex. Science 264:1333-1336.

Dvorak DR, Bishop LG, and Eckert HE (1975) On the identification of movement detectors in the fly optic lobe. J. Comp. Physiol. 100:5-23.

Egelhaaf $M$ (1985) On the neuronal basis of figure-ground discrimination by relative motion in the visual system of the fly. I. Behavioral constraints imposed on the neuronal network and the role of the optomotor system. Biol. Cybern. 52:123-140.
Egelhaaf M, Borst A, and Reichardt W (1989) Computational structure of a biological motion detection system as revealed by local detector analysis in the fly visual system. J. Opt. Soc. Am. A 6:1070-1087.

Egelhaaf M, Borst A, and Pilz B (1990) The role of GABA in detecting visual motion. Brain Res. 509:156-160.

Egelhaaf M and Borst A (1989) Transient and steady-state response properties of movement detectors. J. Opt. Soc. Am. A 6:116-127. Erata: J. Opt. Soc. Am. A 7:172.

Egelhaaf $M$ and Borst A (1993) Movement detection in arthropods. In: FA Miles and $J$ Wallman, eds. Visual motion and its role in the stabilization of gaze. Amsterdam, London, New York, Tokyo: Elsevier, pp. 53-77.

Geiger G and Nässel DR (1981) Visual orientation behavior of flies after selective laser beam ablation of interneurones. Nature 293:398-399.

Geiger G and Nässel DR (1982) Visual processing of moving single objects and wide-field patterns in flies: behavioral analysis after laser-surgical removal of interneurons. Biol. Cybern. 44:141-149.

Gilbert $C$ (1991) Membrane conductance changes associated with the response of motion sensitive insect visual neurons. $Z$. Naturforsch. $45 \mathrm{c}: 1222-1224$.

Götz KG (1972) Principles of optomotor reactions in insects. Bibl. Ophthal. 82:251-259.

Haag J, Egelhaaf J, and Borst A (1992) Dendritic integration of motion information in visual interneurons of the blowfly. Neurosci. Lett. 140:173-176.

Haag J, Egelhaaf M, and Borst A (1993) Active membrane properties of motion-sensitive neurons of the fly Calliphora erythrocephala. In: N Elsner and M. Heisenberg, eds. Gene-Brain-Behavior. Proceedings of the 21 th Göttingen Neurobiology Conference. p. 96.

Haag $J$ and Borst A (1994) Membrane parameters and potential spread in fly lobula-plate neurons. In: $\mathrm{N}$ Elsner and $\mathrm{H}$ Breer, eds. Göttingen Neurobiology Report 1994. Stuttgart, New York: Georg Thieme, p. 447.

Hausen K (1976) Functional characterization and anatomical identification of motion sensitive neurons in the lobula plate of the blowfly Calliphora erythrocephala. Z. Naturforsch. 31c:629-633.

Hausen K, Wolburg-Buchholz K, and Ribi WA (1980) The synaptic organization of visual interneurons in the lobula complex of flies. Cell Tissue Res. 208:371-387.

Hausen K, (1982) Motion sensitive interneurons in the optomotor system of the fly. II. The Horizontal Cells: Receptive field organization and response characteristics. Biol. Cybern. 46:67-79.

Hausen K and Wehrhahn C (1983) Microsurgical lesion of horizontal cells changes optomotor yaw response in the blowfly Calliphora erythocephala. Proc. R. Soc. Lond. B 219:211-216.

Hausen $\mathrm{K}$ and Wehrhahn $C$ (1990) Neural circuits mediating visual flight control in flies. II. Separation of two control systems by microsurgical brain lesions. J. Neurosci. 10:351-360.

Heisenberg M, Wonneberger R, and Wolf R (1978) Optomotor-blind (H31) - a Drosophila mutant of the lobula plate giant neurons. J. Comp. Physiol. 124:287-296.

Hengstenberg R (1982) Common visual response properties of giant vertical cells in the lobula plate of the blowfly Calliphora. J. Comp. Physiol. A 149:179-193.

Hengstenberg R, Hausen K, and Hengstenberg B (1982) The number and structure of giant vertical cells (VS) in the lobula plate of the blowfly Calliphora erytrocephala. J. Comp. Physiol. A 149:163177. 
Laurent G (1993) A dendritic gain control mechanism in axonless neurons of the locust, Schistocerca americana. J. Physiol. $470: 45-54$.

Pierantoni R (1976) A look into the cock-pit of the fly. Cell Tissue Res. 171:101-122.

Poggio T, Reichardt W, and Hausen K (1981) A neural circuitry for relative movement discrimination by the visual system of the fly. Naturwiss 443:446.

Reichardt W (1961) Autocorrelation, a principle for the evaluation of sensory information by the central nervous system. In: WA Rosenblith, ed. Sensory Communication. New York, London: The MIT Press and John Wiley \& Sons, pp. 303-317.
Reichardt W, Poggio T, and Hausen K (1983) Figure-Ground discrimination by relative movement in the visual system of the fly. Part II: Towards the neural circuitry. Biol. Cybern. 46:1-30.

Reichardt W (1987) Evaluation of optical motion information by movement detectors. J. Comp. Physiol. A 161:533-547.

Schmid A and Bülthoff $\mathbf{H}$ (1988) Using neuropharmacology to distinguish between excitatory and inhibitory movement detection mechanisms in the fly Calliphora erythrocephala. Biol. Cybern. 59:71-80.

Segev I, Fleshman JW, and Burke RE (1989) Compartmental models of complex neurons. In: C Koch and I Segev, eds. Methods in neuronal modeling: From synapses to networks. Cambridge, London: MIT Press, pp. 63-96. 